\title{
An educational simulation in construction: the virtual construction simulator
}

Conference or Workshop Item

Accepted Version

Nikolic, D., Jaruhar, S. and Messner, J. I. (2012) An educational simulation in construction: the virtual construction simulator. In: International Workshop on Computing in Civil Engineering 2009, June 24-27 2009, Austin, Texas, pp. 64-72. Available at https://centaur.reading.ac.uk/95262/

It is advisable to refer to the publisher's version if you intend to cite from the work. See Guidance on citing.

Published version at: https://ascelibrary.org/doi/10.1061/41052\%28346\%2963

All outputs in CentAUR are protected by Intellectual Property Rights law, including copyright law. Copyright and IPR is retained by the creators or other copyright holders. Terms and conditions for use of this material are defined in the End User Agreement.

\section{www.reading.ac.uk/centaur}

\section{CentAUR}

Central Archive at the University of Reading

Reading's research outputs online 


\title{
An Educational Simulation in Construction: The Virtual Construction Simulator
}

\author{
Dragana Nikolic ${ }^{1}$, Shrimant Jaruhar ${ }^{2}$, and John I. Messner ${ }^{3}$
}

\begin{abstract}
Students involved in the architecture, engineering and construction disciplines are often faced with the challenge of visualizing and understanding the complex spatial and temporal relationships involved in designing three-dimensional structures. A difficulty inherent in engineering education lies in teaching students decision-making skills, the logic of project management concepts, and the related risks involved in executing complex engineering projects. An evolving body of research applies the use of educational computer simulations to enhance student learning experiences through testing real-world scenarios to aid the development of student decision-making skills. This paper introduces ongoing research at The Pennsylvania State University aimed at improving engineering education in building and construction through interactive construction project learning applications in an immersive virtual reality environment. This research focuses on challenges in understanding and creating construction schedules. The construction industry increasingly employs 4D CAD models for detailed schedule reviews, but commercial applications currently used for creating these 4D models are often inadequate for construction engineering education due to their inability to concurrently create and review schedules. This paper describes the development of a first and second generation Virtual Construction Simulator (VCS), a tool that enables students to create and review construction schedules simultaneously through 3D model interaction. The educational value and utility of the VCS was assessed through surveys, focused group interviews, and a student exercise conducted in a construction management class at Penn State. The results revealed the overall value of the VCS as an effective 4D model creation and schedule review application that fosters collaborative work and greater task focus. In addition, this paper also discusses further development of the VCS educational simulation tool and future research.
\end{abstract}

\footnotetext{
${ }^{1}$ Graduate Research Assistant, Department of Architectural Engineering, The Pennsylvania State University, University Park, PA, dragana@psu.edu.

${ }^{2}$ Former Graduate Research Assistant, Department of Architectural Engineering Department, The Pennsylvania State University, University Park, PA.

${ }^{3}$ Associate Professor of Architectural Engineering, The Pennsylvania State University, University Park, PA, jmessner@engr.psu.edu.
} 


\section{Introduction}

The ability to visualize the built environment and understand building construction processes is critical for students in the architecture, engineering and construction disciplines. Students are often challenged to visualize three-dimensional structures and understand the complex spatial and temporal relationships related to building these structures. In addition to understanding built environment and construction processes, another great challenge in engineering education is teaching students decision-making skills in project management along with the inherent risks involved in designing and building complex structures. Construction processes are becoming increasingly more challenging as project complexity increases and the length of project schedules decreases. Traditional educational approaches to teaching students construction processes have relied on field trips as critical learning experiences and exposure to actual construction settings and situations. However, this approach is often hindered by logistics and is insufficient in duration for students to gain exposure to the various construction stages and gain a deeper understanding of the multiple facets of a building project.

\section{Background}

The acquisition of visualization skills is an important aspect in the education of young engineers. Construction planning requires the development of these skills in order to make informed decisions regarding construction strategies, such as assembly sequences, construction method selection and resource allocation for the successful implementation and the delivery of the project. Teaching students processes and decision making skills in construction planning is particularly challenging due to the complex nature of the detailed knowledge required to thoroughly understand the methods and procedures used to construct a building, along with the impacts of risk and uncertainty on the construction process. Traditionally, 2D drawings have been the dominant method of conveying design ideas and concepts. However, because this representation medium lacks spatial and temporal data, students require additional effort in translating 2D information into three-dimensional space, and there are inherent inefficiencies and inaccuracies related to this mental process (Johnson 1997). Typically, when students learn to develop a construction schedule they rely on their interpretation of 2D drawings to identify activities and decide on a logical sequence. This can pose a significant cognitive load as students are forced to visualize the entire construction process which can be further hindered by a lack of experience in construction schedule management.

Recent research has demonstrated several innovative attempts aimed at bringing real construction site experience into the classroom, such as CALVisual (Bouchlaghem et al., 2002). Through the use of multimedia technologies, this initiative seeks to bring the experience of a construction site into the classroom by building a construction image database (ibid, 2002). Virtual reality (VR) has become an increasingly valued technology that offers students the opportunity to visualize and explore 3D information and data in a dynamic, real-time environment. At Penn State, our students are encouraged to engage in exploration of design and construction processes 
in the Immersive Construction (ICon) $\mathrm{Lab}^{4}$ on a large three-screen stereoscopic display to facilitate better visualization and evaluation of building design and construction processes.

Several current research initiatives in engineering education focus on developing methods to overcome the limitations of employing traditional 2D documents in teaching concepts such as scheduling, site congestion, trades coordination and other project-related construction issues. The growing trend of combining 3D models with construction schedules to create 4D models begins to address the problem of construction process visualization. Because 4D models provide spatial, sequential and temporal information they are valued as tools for effective visualization of construction processes and problem analysis among project participants (Haque 2007). Given that hands-on experience, though highly beneficial, is difficult to incorporate into courses due to costs, safety, and availability, the research focus has now shifted to explore the value of educational simulations as opportunities to experience simulated construction scenarios that closely resemble real construction situations (Sawhney et al. 2000). 4D construction simulation is one aspect of these simulation technologies that provides the educational opportunity to place learning initiatives and control firmly within the grasp of the student. This process reflects the "constructivist learning" paradigm that promotes self-learning in students who perform complicated tasks placed in closely realistic contexts and apply their knowledge and skills in successful task execution (Brooks 1999).

\section{Development of the Virtual Construction Simulator}

The Virtual Construction Simulator research project at Penn State addresses several limitations in visualizing and understanding construction schedule processes. The critical path method (CPM) has been widely employed in teaching construction scheduling. However, as project complexity increases, the CPM method, represented as network diagrams or bar charts, can impose a challenge for students to visualize and understand construction processes due to the amount of information and the interdependence of activities embedded within these processes. This challenge further impedes students' ability to visually understand the logic of construction and develop alternative solutions to construction project issues, such as construction method selection, activity sequencing, activity durations, and temporary facility locations. 4D modeling technology has become widely implemented in the architecture, engineering and construction fields for its ability to facilitate communication between project teams related to visualization of construction documents, identification of potential conflicts, safety issues and other potential challenges (Koo et al. 2000).

Former Penn State graduate student Grace Wang developed the Virtual Construction Simulator (VCS) at Penn State as an educational module using the Deep Creator game engine for improving knowledge in sequencing (Wang 2007; Wang et al 2007). The VCS project focuses on investigating the effectiveness of using an interactive 4D educational simulation application for construction schedule creation and allows for expansion of its functionality to other construction concepts. VCS was developed as

\footnotetext{
${ }^{4}$ http://www.engr.psu.edu/ae/cic/facilities/icon/
} 
an alternative approach to generating 4D models compared to most readily available 4D applications. In 2005, at the start of this project, commercially available 4D applications functioned primarily as schedule review tools, not schedule development tools in which visualizing the $3 \mathrm{D}$ model and creating the schedule were separate processes carried out in different applications. One major limitation to this process is that the schedule and the 3D model are seen as separate inputs that are subsequently linked to create a 4D model output [Figure 1 left]. Conversely, the VCS approach makes both creating schedules and reviewing 3D information integral parts of developing 4D models [Figure 1 right]. The VCS application allows students to interact with a 3D model by creating groups of individual objects, attaching activities to groups, and generating sequences between these activities [Figure 2 left]. Thus, the VCS approach generates a construction schedule directly from a 3D model, eliminating the need for a CPM schedule.

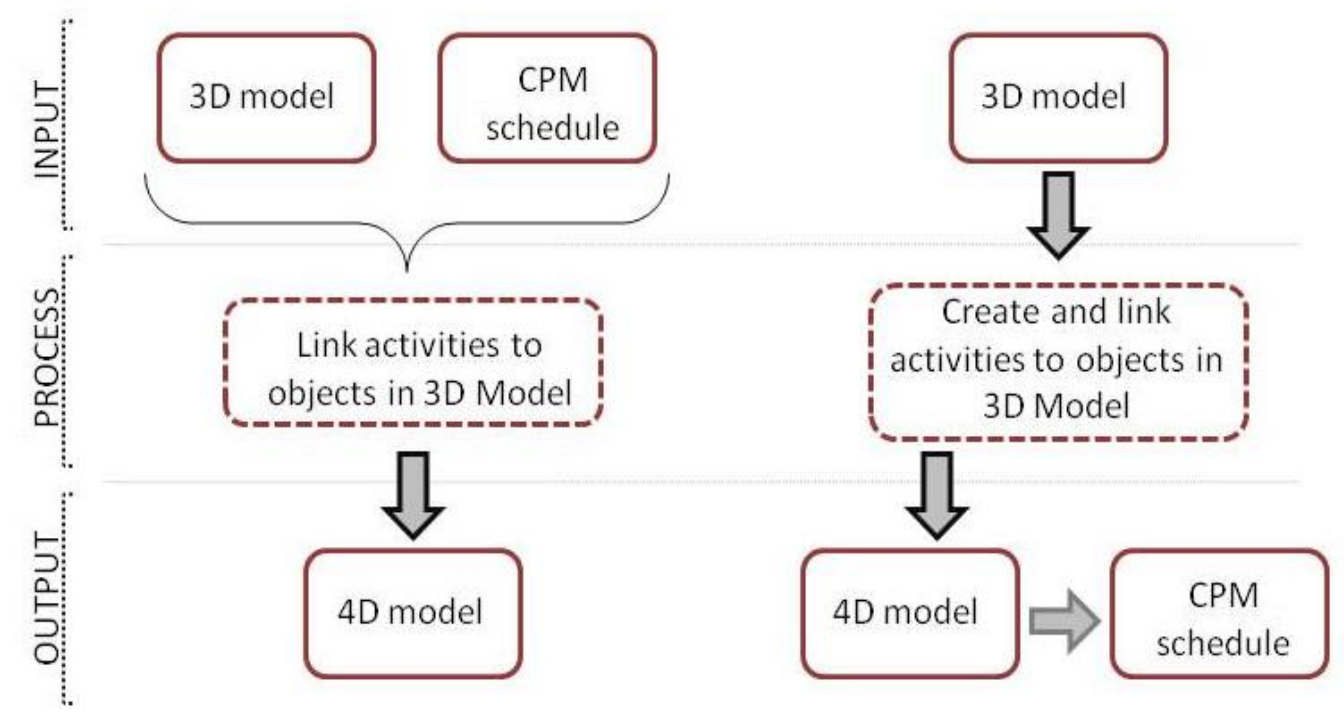

Figure 1 Left: Traditional approach to $4 D$ modeling with a CPM schedule as an input; Right: VCS approach to $4 D$ modeling with a CPM schedule as an output.

In Fall 2006 Wang performed an experiment by examining time spent on different stages of sequencing and communication efficiency. This experiment was developed around a case study, the MGM Grand Hotel in Las Vegas, Nevada, particularly because its precast concrete structural system and placement in an actual context provided students with a comprehensive view of construction processes and related details. Using two different applications - the VCS and the commercial 4D modeling application; ten groups of 3-4 randomly assigned students in an upper level construction class were asked to develop a Short Interval Production Schedule (SIPS) for one hotel floor and its building components, including walls, floors, beams, frames, and structural connections. The study compared two sets of five groups, one that employed the VCS and another that used MS Project and NavisWorks, to identify 
variations in sequencing construction processes. Each group was allotted four lab session hours and were audio and video recorded for later analysis. Figure 2 (right) shows a group working through the lab using the VCS.
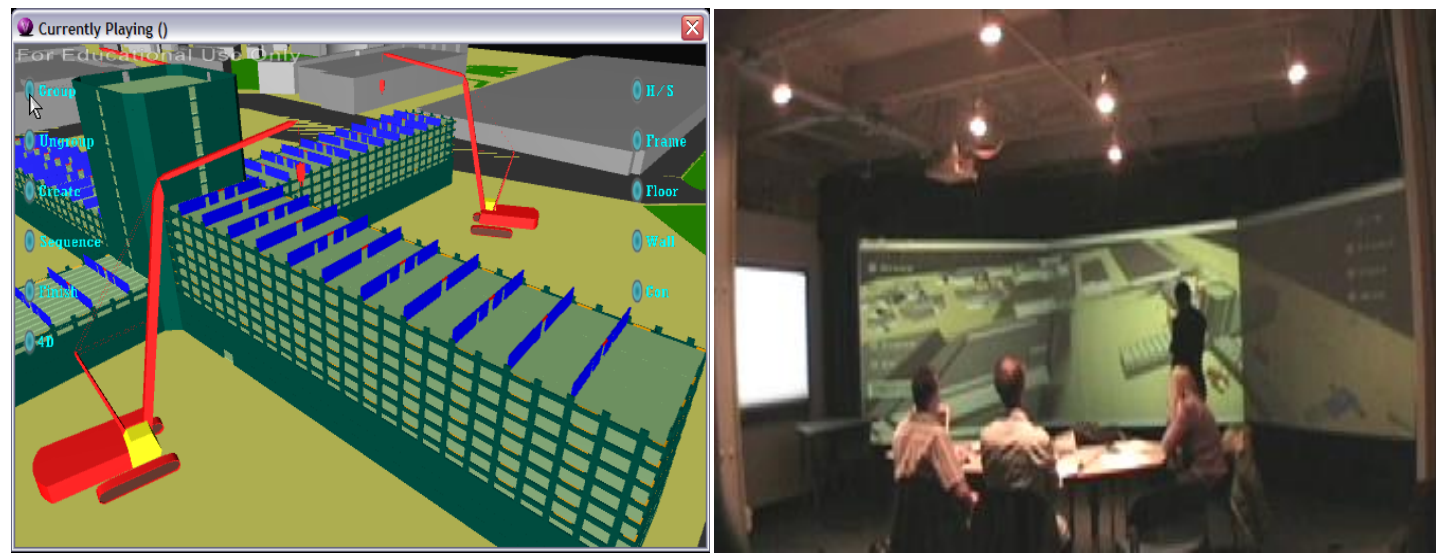

Figure 2 Left: VCS 1 User Interface; Right: student group in the Immersive Construction Lab using the VCS 1 during the working session.

Data collection included surveys, focus groups, and direct laboratory observations recorded on videos that were later analyzed with Studiocode ${ }^{5}$ video analysis software. Analysis showed that by using the VCS approach students spent less time trying to understand construction problems and more time analyzing and creating appropriate construction sequences (Table 1) and that students who worked with the VCS used allotted time more effectively for communication. However, the results also showed that schedule quality, although slightly improved for groups using the VCS, was not significantly different from groups that used commercial 4D CAD applications.

Table 1: Percentage of Time Spent by Communication Categories for Video Duration

\begin{tabular}{ccc} 
& $\begin{array}{c}\text { Experimental Group } \\
\text { (VCS) }\end{array}$ & $\begin{array}{c}\text { Control Group } \\
\text { (NavisWorks) }\end{array}$ \\
\hline Goal Clarification & $5.09 \%$ & $4.53 \%$ \\
Solution Generation & $17.84 \%$ & $11.12 \%$ \\
Analysis & $65.73 \%$ & $79.58 \%$ \\
Evaluation & $7.92 \%$ & $3.13 \%$ \\
Decision & $3.42 \%$ & $1.64 \%$ \\
\hline
\end{tabular}

\footnotetext{
${ }^{5}$ http://www.studiocodegroup.com/Studiocode\%20Education.htm
} 


\subsection{Virtual Construction Simulator II}

Building upon Wang's study, a second version of the VCS was developed in 2007 using the Irrlicht ${ }^{6}$ open source rendering engine. This second version, called VCS II, solves several scalability issues with the initial application, improves the user interface and functionality, and addresses the database implementation limitations in the initial version. Specific VCS I limitations included the requirement that each three-dimensional element is LISP coded, which limited the ability to easily develop new simulations with other 3D models. A dedicated database to store groups, activities and sequence data through a Microsoft SQL server was also used, which limited the ability to easily distribute the application. For those reasons, Irrlicht was evaluated as an alternative to the Deep Creator game engine, allowing for greater flexibility in expanding the functionality of the VCS by using a more common C++ language as well as facilitating free dissemination of the simulation. Based on the assessment of VCS I, new features added to VCS II included preset viewpoints, sequencing activities in a chain, automatic schedule generation, and ability to save and load work at any point [Figure 3]. As an output, VCS II generates an XML file containing the construction schedule that can be reloaded at any time.
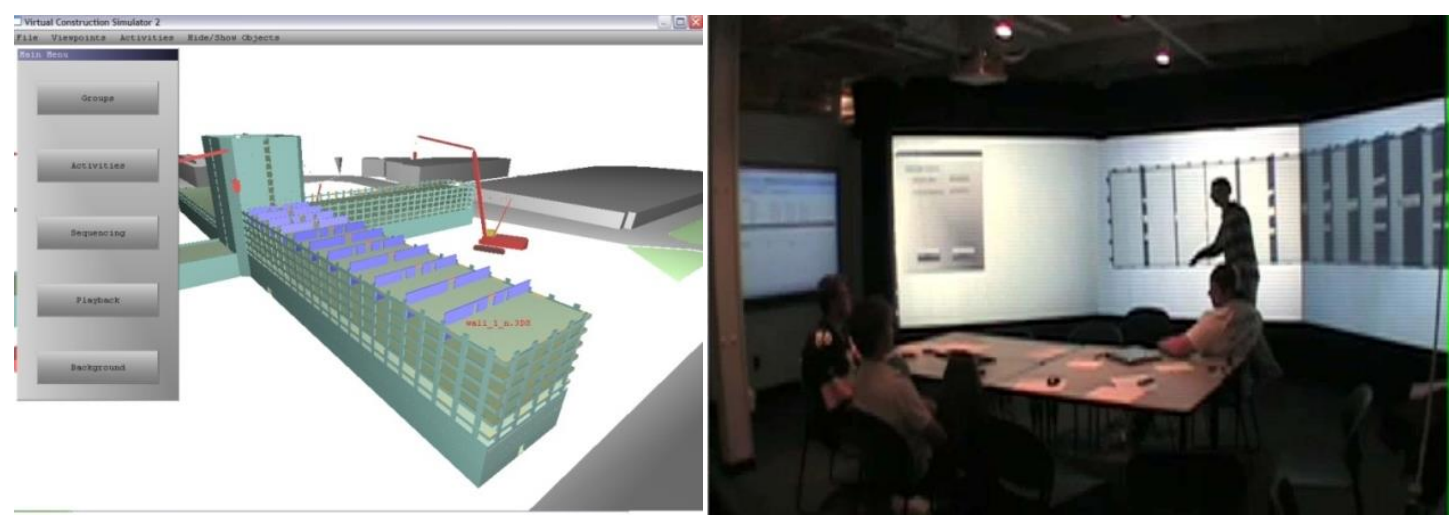

Figure 3: Left: VCS II User Interface; Right: Students using VCS II in the ICon Lab

VCS II was implemented in Fall 2007 in the same engineering course as the previous study with an improved interface for entering activities and sequencing data. Research was again done to evaluate the use of VCS II using the same experiment method of 10 groups of 3-4 randomly assigned students who were asked to develop a schedule for one floor of the same MGM Grand Hotel. The students had to sequence the erection of precast floor slabs, walls, frames, beams and the connections between the frames and the slabs for one floor of the hotel wing. Data collection was again comprised of surveys, focus groups, and direct observations that were recorded for later comparison. This survey data was used to compare the VCS I and VCS II

\footnotetext{
${ }^{6} \mathrm{http} / / /$ irrlicht.sourceforge.net/downloads.html
} 
application interfaces as well as the value in schedule generation of each version. Tests of discussion comprehensiveness were done by comparing audio and video recordings from both the 2006 and 2007 studies to determine if any changes to the assignment were needed. Groups using the first and the second version of the VCS were compared in two main categories: interaction with the application and team discussion. Application interaction was analyzed by examining activities such as navigation, authoring the content, and reviewing and analyzing the outcomes. The team discussion analysis focused on time spent by each member discussing the task, as well as the time a facilitator spent assisting the team.

\subsection{Results}

The comparison of the survey results from both studies revealed students' perception of the VCS II interface as 'logical and smooth' and 'easy to use' (Jaruhar, 2007). Students demonstrated greater confidence in their scheduling work after the exercise and generally asserted that 4D modeling was valuable as a communication tool, and that it helped their understanding of the SIPS schedule.

Video analysis concluded that the second version of the software was more intuitive and user-friendly and that the time the facilitator needed to assist groups decreased significantly [Table 2]. The discussion time among group members varied depending on the VCS version used. The average time groups spent in discussion was almost twice as much as for the first version of the VCS (11 min) compared to the second (6 min), indicating a different group dynamic in discussing the problem. Further analysis of discussion comprehensiveness indicated that students using the VCS I engaged in more detailed discussions of relevant items related to the construction schedule and 4D model. It was apparent that the teams using the VCS I spent more time performing calculations and discussing the construction sequence before authoring the content, whereas groups using the VCS II performed calculations and preparations prior to the lab session and moved directly into planning the schedule for the simulation (Leicht et al. 2008). Thus, the results illustrate a significant decrease in the time that student groups spent working on the exercise, but also a slight decrease in the quality of the schedule output and the average project grade (a 3\% reduction on average on a 100\% scale). One possible factor that may have influenced this performance differential is the time of the first study (October) versus that of the second (November). Further analysis remains to be performed on student performance and understanding of construction processes using the VCS. Focus group interviews confirmed the value of the VCS II as a visualization tool and provided some suggestions for the further improvement of the interface and features. 
Table 2. The comparison of the time spent using the VCS 1 and VCS 2 by each group, in minutes and as percentage of the total interaction time with the software

\begin{tabular}{lcccccccc}
\multicolumn{1}{c}{ VCS 1 } \\
\hline & Group 1 & Group 2 & Group 3 & Average & Group 4 & Group 5 & Group 6 & Average \\
Navigating & 7.87 & 13.02 & 14.71 & 11.87 & 41.02 & 25.3 & 20.15 & 28.82 \\
the model & $(6.6 \%)$ & $(17.2 \%)$ & $(19.7 \%)$ & $(13.2 \%)$ & $(60.0 \%)$ & $(77.3 \%)$ & $(63.8 \%)$ & $(65.2 \%)$ \\
\hline Authoring & 108.93 & 59.57 & 52.61 & 73.70 & 18.58 & 7.21 & 9.67 & 11.82 \\
Content & $(90.9 \%)$ & $(78.7 \%)$ & $(70.5 \%)$ & $(81.8 \%)$ & $(27.2 \%)$ & $(22.0 \%)$ & $(30.6 \%)$ & $(26.7 \%)$ \\
\hline $\begin{array}{l}\text { Analyzing } \\
\text { outcome }\end{array}$ & 3.06 & 3.14 & 7.33 & 4.51 & 8.81 & 0.21 & 1.75 & 3.59 \\
\hline $\begin{array}{l}\text { Total } \\
\text { interaction } \\
\text { time with } \\
\text { the software }\end{array}$ & $11.6 \%)$ & $(4.1 \%)$ & $(9.8 \%)$ & $(5.0 \%)$ & $(12.9 \%)$ & $(0.6 \%)$ & $(5.5 \%)$ & $(8.1 \%)$ \\
\hline
\end{tabular}

\section{Limitations and Future Development}

Current user interface limitations of the VCS II to be addressed include pre-set viewpoints, visual appearance, and lack of features such as clipping planes that would facilitate sequencing and visualization of interior construction activities. Because the use of the MGM Grand Hotel as a simulation focused only on one floor, the detection of greater variations in the use of the VCS and the traditional 4D modeling applications may have been limited. The use of additional case studies would reveal the suitability of the VCS for more complex schedule development. Both versions of the simulator, however, provide a platform for future development and expansion of content and functionality for an enriched learning experience. VCS allows students to visually sequence project activities through direct interaction with a model and simultaneous processes of creating and reviewing a construction schedule. However, student understanding of the logic in creating a schedule and testing possible solutions for given project characteristics remains an activity for class review and instructor feedback. The next VCS development step will provide means for giving students feedback to support their understanding of construction concepts through testing hypotheses and learning through trial and error.

The Virtual Construction Simulator has demonstrated an improvement on the process of creating, reviewing and visualizing construction schedules. Nevertheless, as a 4D schedule planning application, the VCS represents only the first step in the development of a more comprehensive construction simulator to provide specific contextual project information and allow students to experience the construction process as projects virtually progress. A major limitation of the VCS is that no specific project-based constraints exist that would motivate consideration of the most feasible construction sequence. The overall objective is to extend the functionality of the Virtual Construction Simulator to allow for a scenario based, student-centered learning experience through practicing decision-making skills, testing hypotheses, and exploring the consequences of decision making through trial and error. This approach aims to provide students with the ability to explore construction options, 
understand how to optimize construction processes and apply this knowledge in reallife situations.

\section{Conclusion}

The construction industry is a rapidly evolving field that poses challenges to educators to prepare students with appropriate and necessary skills. This paper discussed ongoing research at Penn State aimed at improving engineering education in building and construction through the use of interactive construction project learning applications in an immersive virtual reality environment. The research focused on investigating the effectiveness of 4D modeling applications for schedule visualization in construction engineering education. The development of the Virtual Construction Simulator has sought to address challenges in developing construction schedules by allowing students to directly interact with $3 \mathrm{D}$ data. To assess the value of the VCS, two studies were conducted. The first study compared the VCS learning module with a more traditional 4D modeling process; the second assessed the differences in usability of two VCS versions. Overall, the added interactivity in the VCS helped students in developing higher-quality schedules and also provided an enjoyable learning experience. The VCS implementation has been proven to encourage collaborative group work, engage students and foster greater solution generation through better visualization of construction processes.

The contribution of this research is the development of an open source educational application intended for dissemination to other educational facilities. Documentation of the usability of the VCS, along with lessons learned, will serve to create guidelines for future development of educational simulations.

The next research step is to extend the functionality of the VCS and add project-based constraints that will allow students to explore possibilities and consequences of decision making, evaluate construction options, understand how to optimize construction processes, and build confidence in managing similar situations in reality.

\section{Acknowledgements}

We would like to thank VCS authors (Grace Wang and Shrimant Jaruhar), as well as the AE student participants for agreeing to be observed and recorded during the study presented in this paper. The authors thank the National Science Foundation (Grant 0343861 and 0348457) for support of this project. Any opinions, findings, conclusions, or recommendations expressed in this paper are those of the authors and do not necessarily reflect the views of the National Science Foundation.

\section{REFERENCES}

Bouchlaghem, N., Wilson, A., Beacham, N. and Sher, W. (2002) Computer Imagery and Visualization in Built Environment Education: The CAL-Visual Approach Innovations in Education and Teaching International, Vol 39-3

Brooks, J. G., (1999) In search for understanding: The case for constructivist classrooms. Alexandria, VA; 1999 
Corti, K., (2006) Games-based Learning; a serious business application, Retrieved from: http://www.pixelearning.com/docs/seriousgamesbusinessapplications.pdf

Gee, J.P., (2003) What video games have to teach us about learning and literacy Computers in Entertainment (CIE), Vol. 1, Issue 1

Haque, E.M., (2007) n-D Virtual Environment in Construction Education, The 2nd International Conference on Virtual Learning, ICVL 2007

Jaruhar, S. (2007) Development of Interactive Simulations for Construction Engineering Education. Master's Thesis, The Pennsylvania State University

Johnson, S. (1997) What's in a Representation, Why Do We Care, and What Does it Mean? Examining the Evidence from Psychology, Proceedings of ACADIA '97 Conference, Cincinnati, Ohio, October 3-5

Koo, B., and Fischer, M. (2000). "Feasibility study of 4D CAD in commercial construction." Journal of Construction Engineering and Management, ASCE, 126(4), 251260 .

Leicht, R.M., Hunter, S.T., Saluja, C. and Messner, J.I. (2008) Methodology for Utilizing Observational Studies to Perform Research on Construction Teams, Work in progress, Penn State University, 2008

Mukherjee, A. and Rojas, E.M (2003) Applying Multi Agents to General Purpose Situational Simulations in Construction Management, 2003, Retrieved from: http://www.hitl.washington.edu/publications//r-2003-21/r-2003-21.pdf

Prensky, M. (2000) Digital Game-Based Learning; McGraw-Hill Companies

Sawhney, A., Koczenasz, B., Bashford, J., and Mund, A., (2000) Internet-Based Interactive Construction Management Learning System. Proceedings of the 2000 American Society for Engineering Education Annual Conference \& Exposition, St. Louis, Missouri

Wang, L. and Messner, J.I. (2007) Virtual Construction Simulator: A 4D CAD Model Generation Prototype. ASCE Workshop on Computing in Civil Engineering Pittsburgh, PA, 2007

Wang, L. (2007) Using 4D Modeling to Advance Construction Schedule Visualization in Engineering Education. Master's Thesis, The Pennsylvania State University 